\title{
Exploiting Stability Regions for Online Signature Verification
}

\author{
Antonio Parziale, Salvatore G. Fuschetto, and Angelo Marcelli \\ Natural Computation Laboratory, \\ DIEM, University of Salerno \\ Via Ponte Don Melillo, Fisciano (SA), Italy \\ \{anparziale, amarcelli\}@unisa.it, sgfuschetto@gmail.com \\ http://nclab.diiie.unisa.it
}

\begin{abstract}
We present a method for finding the stability regions within a set of genuine signatures and for selecting the most suitable one to be used for online signature verification. The definition of stability region builds upon motor learning and adaptation in handwriting generation, while their selection exploits both their ability to model signing habits and their effectiveness in capturing distinctive features. The stability regions represent the core of a signature verification system whose performance is evaluated on a standard benchmark.
\end{abstract}

Keywords: online signature verification, stability region, handwriting generation, motor learning.

\section{Introduction}

Handwritten signatures are an interesting biometric characteristic, since they are largely used and accepted in daily life as proof of one individual's identity.

As any handwriting, they are obtained by concatenating elementary movements, or strokes, in such a way that their execution requests the minimum amount of metabolic energy. Such an optimization is learned along the years by repeated practice, so that signing becomes automated and can be performed, at least partially, without any proprioceptive feedback, as it was an elementary movement. When the signature has been completely learned, i.e. it becomes a distinctive feature of the subject, it is stored in the brain as a motor plan that incorporates both the sequence of target points, i.e. the points where two successive strokes join, and the sequence of motor command to be executed to draw the desired shape between them [1. The encoding of such a motor plan, moreover, is independent of the actuator $[2]$.

According to those findings, multiple executions of the signature by the subject may produce ink traces with different shapes only because of variations on both the psychophysical conditions of the subject and the signing conditions, since the motor plan remains the same 3.4. It is also expected, however, that the variations in the signing conditions mentioned above may affect only some of the signature features, but not all of them. For this reason, one of the aspects

A. Petrosino, L. Maddalena, P. Pala (Eds.): ICIAP 2013 Workshops, LNCS 8158, pp. 112-121, 2013.

(C) Springer-Verlag Berlin Heidelberg 2013 
around which is centered the research activity in the field of signature verification is the detection of stability regions, which are the traits of ink that are shared by different executions of the signature.

Signature stability can be estimated directly from the signature signal or indirectly on the set of features used for representing the signature [3]. Among the methods for directly estimating on-line signature stability, those using DTW to derive a local stability function [56], are the most similar to the one presented in this paper, in that the analysis of local stability is used to select the best subset of reference signatures.

In this work we present a method for the detection of stability regions in online signatures. The proposed method assumes as stability regions the longest common sequences of similar strokes between pairs of signatures. This assumption follows from the observation that, as discussed above, signing is the automated execution of a well-learned motor task, and therefore repeated executions should produce, at least ideally, the same movements and therefore ink traces with the same shapes. Because of the variations in the signing conditions, it happens that the ink traces are different, but because they results from the execution of the same motor plan, and because the effects of different initial conditions in signing attenuate quickly during writing, it is expected that only short sequences of strokes will exhibit different shapes. The longer the sequence of similar strokes the longer the time during which the same motor program is executed under the same conditions.

Once the stability regions of the genuine signatures have been found, the classification of a questioned signature as either genuine or forged is achieved by evaluating the longest common sequence of similar strokes between the ink trace of the questioned and those of the genuine containing the selected stability regions.

The remaining of the paper is organized as it follows. In section 2 we illustrate the method for finding the stability regions between a pair of signatures, and discuss how they can be used for selecting the reference signatures. The selected reference signatures and their associated stability regions are then used in a signature verification experiment reported in section 3. Eventually, in the conclusion we discuss the performance achieved by the proposed method and outline our future research.

\section{Modeling Stability in Signatures}

In a previous work we have introduced an earlier version of our method for finding stability regions between two signatures [7. Here we introduce a new version of the method, that takes in account the existing correlation between consecutive strokes, and an alternative criterion for finding stability regions in a set of $\mathrm{N}$ signatures. 


\subsection{Searching for Stability Regions between Two Signatures}

The proposed method assumes that the signature signal has been segmented into a sequence of strokes, each of which has been label as ascender, descender or normal, and the detection of the stability regions is achieved by an ink matcher that finds the longest common sequences of strokes with similar shapes between the ink traces of a pair of signatures. For deciding when two sequences are similar enough, i.e. when they match, the method exploits the concept of saliency that has been proposed to account for attentional gaze shift in primate visual system [8. Accordingly, sequence of strokes that are "globally" more similar than other will stand out in the saliency map.

To implement such an approach one needs to define a scale space, to find a similarity measure to be adopted at each scale, to compute the saliency map, and eventually to select the matching pieces of ink. We have adopted as scale the number of strokes in the sequences whose similarity is being measured. Such a number will be referred in the following as the length of the sequence. Let us assume that the two sequences have $\mathrm{N}$ and $\mathrm{M}$ strokes, respectively. The number of scales corresponds to the length $K \leq \min (N, M)$ of the longest common sequence of strokes. Note that the inequality sign holds because we assume that ascenders and descenders can match only themselves, not normal strokes. Thus, $\mathrm{K}$ represents the length of the longest common sequence of compatible strokes, i.e. strokes that can be matched. Successive scales are obtained considering sequences made of $k=K, K-1, \ldots, 2$ strokes [9]. As similarity measure, we adopt the Weighted Edit Distance (WED), which measures the shape similarity between pair of strokes [10. The shape similarity of a sequence is obtained by adding the WED of its strokes. After the shape similarity is evaluated at each scale, we compute its saliency as it follows. At each scale k, the most similar pair of sequence is selected and the saliency $S_{i j}^{k}$ of all its strokes is computed as $S_{i j}^{k}=W E D_{i j} / k$. Thus, the saliency map for a pair of inks made of $\mathrm{N}$ and $\mathrm{M}$ strokes, respectively, assumes the shape of an NxM array, whose elements are either 0 , in case of incompatible strokes, or $\sum_{K} S_{i j}^{k}$. The saliency map is then

thresholded, and the longest diagonal sequences of values $S_{i j}^{k}$ greater than the threshold $S_{t h}$ are selected and they are named invariants.

As previously said, an ink trace is the result of a complex motor task that can be decomposed in elementary movements. It is important to point out that each stroke is not independent by the others but it is affected by the movements realized before it and by those that will be realized after it. It means that another parameter is needed to describing globally a strokes sequence and giving a reliability measure of the similarity evaluated before. For this purpose, given an invariant that goes from stroke $T_{1}$ to stroke $T_{2}$, we introduce another parameter named compatibility:

$$
C=\sum_{t=T_{1}}^{T_{2}} W E D_{t} * \beta_{t}
$$

where $\beta_{t}$ takes into account the difference of slope variation between two consecutive strokes in the reference and in the query signature. 


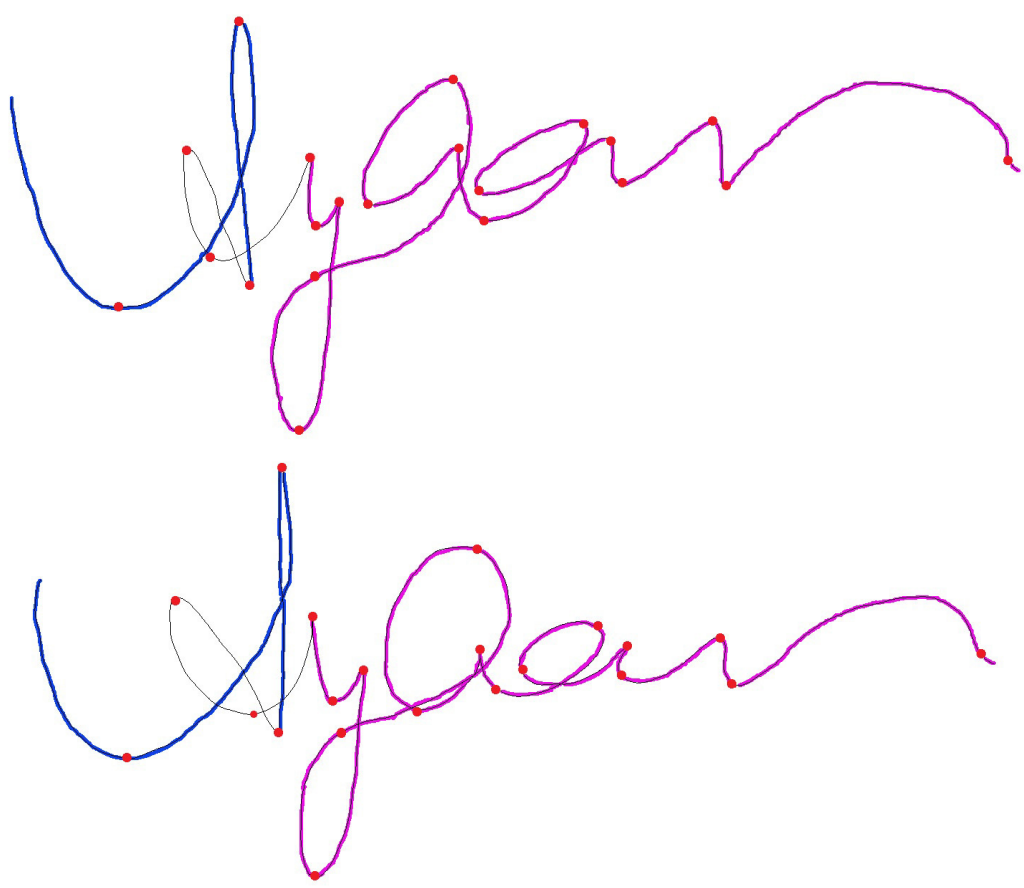

Fig. 1. The segmentation points (dots) and the stability regions (bold) of two signatures

Therefore, the invariants with a compatibility greater than a threshold $C_{t h}$ are the stability regions between pair of signatures, as shown in (Fig. 1)

\subsection{Searching for Stability Regions in a Set of N Signatures}

In way of principle, one would expect that the stability regions appear in all the signatures of a subject, and therefore would define the stability regions of a set of $\mathrm{N}$ signatures as the longest common subsequences of the stability regions computed for every available pair of signatures. In practice, however, both the stroke segmentation and the ink matching may introduce errors in locating the segmentation points and/or in deciding when a sequence of strokes is similar to another, that may produce different stability regions for the set of signatures. For all these reasons, we must define how many and which are the most representatives signatures, hereinafter called references, of a given data set. As with regards to the number of references, we restrict our investigation to 2 and 3 , because this is the number of genuine signatures generally available in real life applications. Therefore, given a set of $\mathrm{N}$ signatures we have $\left(\begin{array}{c}N \\ 2\end{array}\right)$ and $\left(\begin{array}{c}N \\ 3\end{array}\right)$ subsets of signatures respectively, and we need to find the most representative ones. 
According to our basic assumption, longer stability regions correspond to longer sequence of elementary movements executed in a highly automated fashion. Because the level of automation is the result of the learning process described above, and because the learning is an individual feature, long stability regions are more subject-specific than short ones. Accordingly, we can select as references the signatures corresponding to the longest stability regions.

Another suitable criterion is to select as references the signatures that minimize the equal error rate on a verification experiment performed on the training set composed by genuine and forgery samples.

\section{Experimental Validation}

The detection of the stability regions and its effectiveness in a verification problem can be modulated by choosing the thresholds $S_{t h}$ and $C_{t h}$ of the ink matcher stage and the number of references. A set of experiments has therefore been executed for evaluating the effect of the value of those parameters on the performance.

\subsection{The Datasets}

The performance of the proposed method has been evaluated on the signatures of SVC2004 database 11 and on the signatures of the Blind subcorpus of the SUSIG database [12. The first contains 20 genuine and 20 forgery signatures for each of 100 subjects, for a total of 40,000 specimen divided in training set, which is publicly available, and test sets that is not available. The second database contains 10 genuine and 10 forgery signatures for each of 60 subjects. The protocol proposed during the First International Signature Verification Competition [1]. has been adopted during the test:

1. Skilled forgery detection: For each subject the genuine signatures are randomly divided in two disjoined subsets: 5 samples are used together with 5 forgery signature as training set, the remaining are added to the set of forgery signatures and used as test set.

2. Random forgery detection: the training set is built as before, whereas the test set includes as forgery 20 genuine signatures produced by 20 different subjects.

\subsection{The Classification Rule}

In this work we are interested in presenting and evaluating the method for the detection of the stability regions by means of a signature verification experiment. For this reason, we use a simple classification rule for showing that the obtained results mainly depend on the selection of the stability regions and not on the classification scheme. Each signature of the training set, either genuine or not, 
is mapped in a $N$ dimensional space $\Gamma$, where $N$ is the number of references, and whose dimensions are:

$$
r_{i}=\frac{L_{m}\left(f, r e f_{i}\right)}{L_{s}\left(r e f_{i}\right)} \quad i=1, \ldots, N
$$

In equation 2, $L_{m}$ is the length of the longest common sequence of strokes between the signature under verification $f$ and the reference $r e f_{i}$ and $L_{s}$ is the length of the stability region found in $r e f_{i}$. Each $r_{i}$ ranges between 0 and 1 . For the sake of clarity, let's consider the case $N=2$. In such a case, $\Gamma$ assumes the shape of a square, whose vertices are $(0,0),(0,1),(1,0)$ and $(1,1)$. In such a space, genuine signatures should have a (long) match with the stability regions of both references, and therefore should be represented by points close to the vertex of coordinates $(1,1)$. On the contrary, forged signatures should not have a (long) match with any of the stability regions of the references, and therefore should be represented by points near to the vertex of coordinates $(0,0)$. Thus, it is possible to find two thresholds, $T_{g}$ and $T_{f}$, by maximizing both the number of genuine signatures correctly classified whose distances from the vertex $(1,1)$ is smaller than $T_{g}$, and the number of forgery correctly classified whose distances from the vertex $(0,0)$ is smaller than $T_{f}$, as shown in figure 2. Then, the selected references and the value of $T_{g}$ and $T_{f}$ model the signing habit of the subject with respect to a given population of writers. The verification is eventually achieved by mapping the signatures under verification in $\Gamma$ as above, and by computing the distances $d_{f}$ and $d_{g}$ between the point representing the signature and the two vertices $(0,0)$ and $(1,1)$, respectively. The decision criterion follows naturally from above:

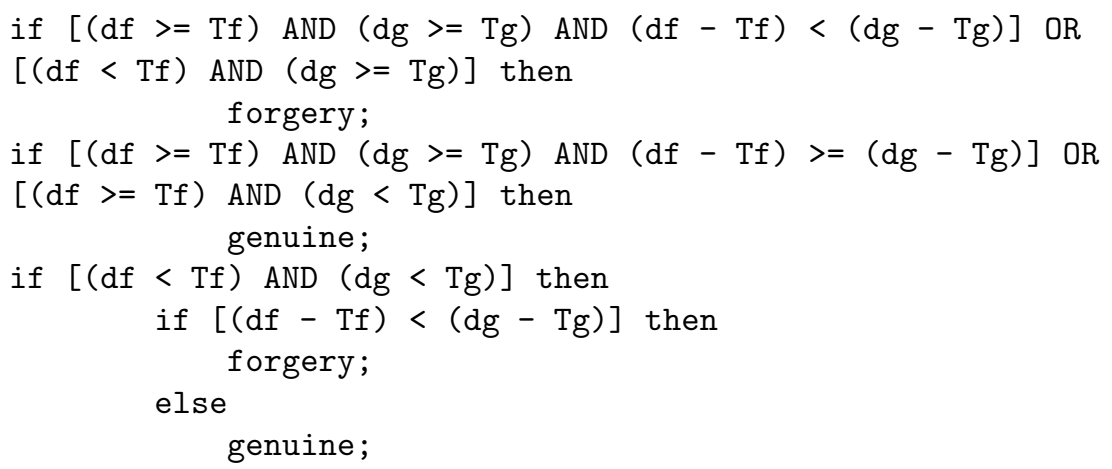

\subsection{Results}

The classification rules are applied for different values of $S_{t h}$ and $C_{t h}$ in four different conditions:

1. ref2_H0: 2 genuine signatures with the longest stability region are selected as references by the training set; 


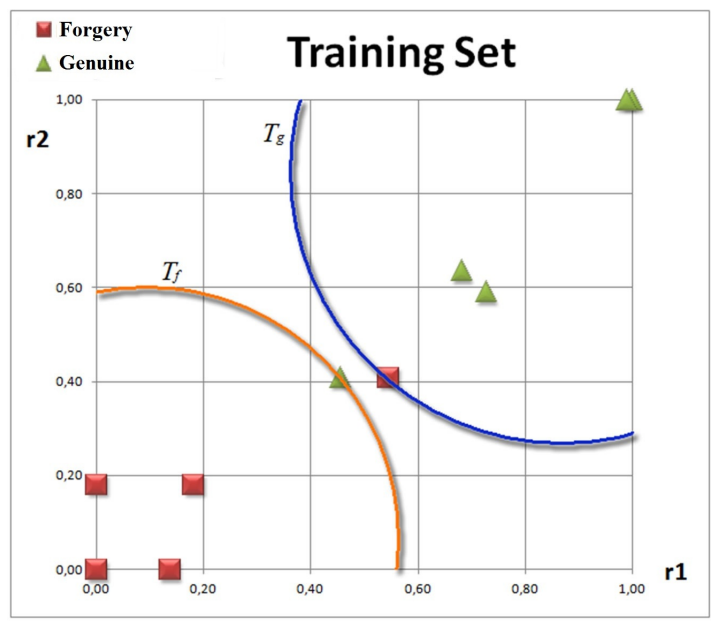

Fig. 2. The signatures from the training set and the decision region for genuine and forgery

2. ref2_H1: 2 genuine signatures that minimize the EER on the training set are selected as references;

3. ref3_H0: 3 genuine signatures with the longest stability region are selected as references by the training set;

4. ref3_H1: 3 genuine signatures that minimize the EER on the training set are selected as references.

In the experiments, $S_{t h}$ and $C_{t h}$ were chosen by using a grid search with $S_{t h}=$ $(0.70 \div 1.0)$ and $C_{t h}=(0.40 \div 0.80)$. In the figures 3 and 4 we plot $E E R_{a v g}$ as function of $C_{t h}$ for $S_{t h}=0.9$ in case when the test set includes skilled and random forgeries, respectively. The best results on the SVC2004 database were obtained using $S_{t h}=0.9, C_{t h}=0.75$ and selecting as references the two genuine signatures that minimize the EER on the training set. The test executed on the SUSig dataset confirmed that the best performance was obtained by selecting the references as above, whereas the best values for the parameters of the ink matcher stage were $S_{t h}=0.9$ and $C_{t h}=0.5$. In this case, a smaller value of compatibility is required because the signatures are shorter and segmented in a smaller number of strokes than the samples of the SVC2004 database and then shorter stability regions are obtained. Table 1 reports the performance of our method in its best configuration in comparison with those participating in the SVC2004 benchmark.

\section{Conclusions}

We have presented a model for describing the signature of a subject centered upon the concept of the stability regions. In particular, we have discussed about 


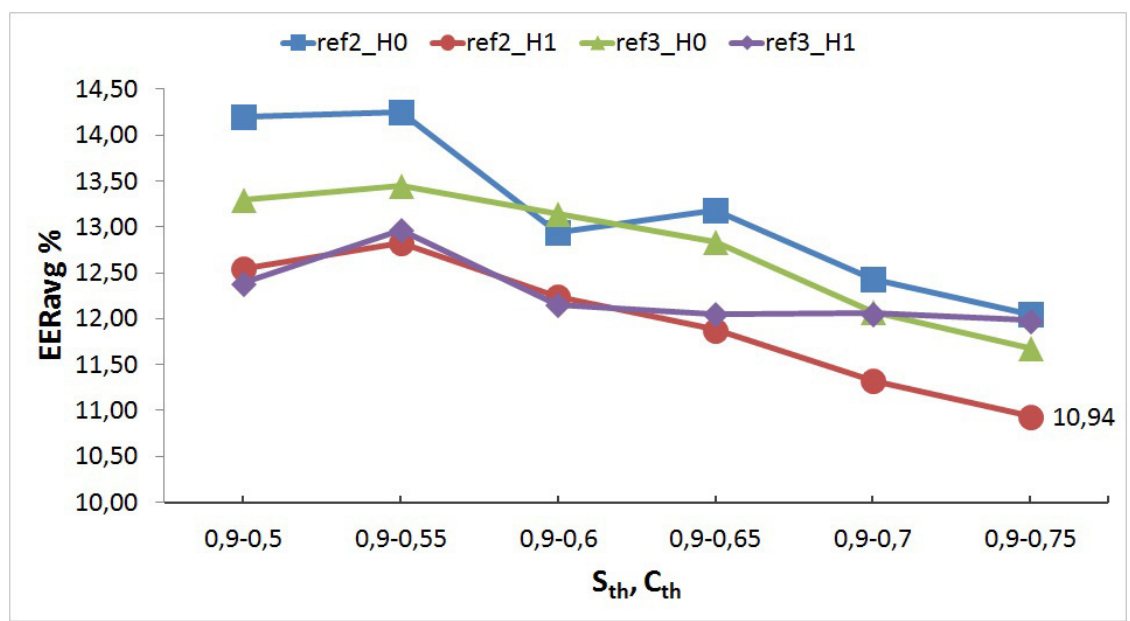

Fig. 3. Trials performed on the test set composed by genuine and skilled forgery signatures. $E E R_{a v g}$ values are obtained with different scheme of references selection and different values of ink matching threshold $S_{t h} C_{t h}$.

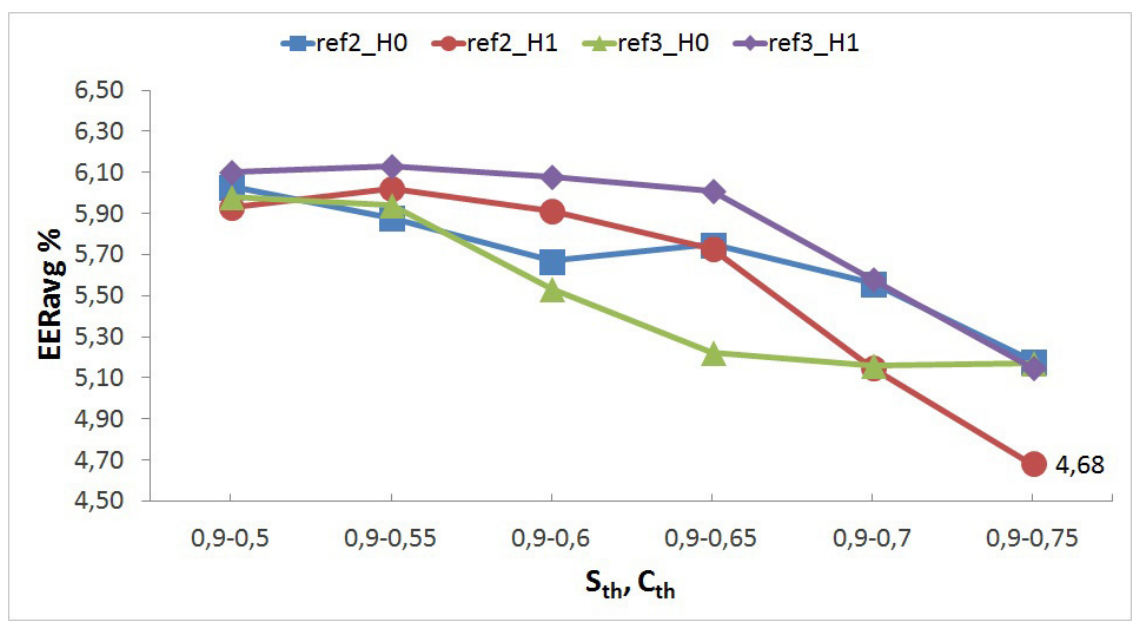

Fig. 4. Trials performed on the test set composed by genuine and random forgery signatures. $E E R_{\text {avg }}$ values are obtained with different scheme of references selection and different values of ink matching threshold $S_{t h} C_{t h}$. 
Table 1. Results on SVC2004 database

\begin{tabular}{|c|c|c|c|c|}
\hline \multirow{2}{*}{ Team ID } & \multicolumn{2}{|c|}{ skilled forgeries } & \multicolumn{2}{c|}{ random forgeries } \\
\cline { 2 - 5 } & EERavg & EERsd & EERavg & EERsd \\
\hline 106 & $5.50 \%$ & $7.73 \%$ & $3.65 \%$ & $4.80 \%$ \\
126 & $6.45 \%$ & $10.41 \%$ & $3.49 \%$ & $4.53 \%$ \\
124 & $7.33 \%$ & $7.71 \%$ & $2.93 \%$ & $3.72 \%$ \\
115 & $9.80 \%$ & $13.90 \%$ & $2.90 \%$ & $3.60 \%$ \\
Our method & $\mathbf{1 0 . 9 4 \%}$ & $\mathbf{2 . 9 7 \%}$ & $\mathbf{4 . 6 8 \%}$ & $\mathbf{2 . 3 8 \%}$ \\
114 & $11.10 \%$ & $11.11 \%$ & $3.36 \%$ & $4.36 \%$ \\
$119 \mathrm{c}$ & $11.98 \%$ & $17.65 \%$ & $2.87 \%$ & $3.68 \%$ \\
$119 \mathrm{~b}$ & $11.99 \%$ & $17.66 \%$ & $2.88 \%$ & $3.68 \%$ \\
118 & $14.34 \%$ & $16.11 \%$ & $4.29 \%$ & $5.45 \%$ \\
$119 \mathrm{a}$ & $14.91 \%$ & $18.98 \%$ & $2.90 \%$ & $3.64 \%$ \\
116 & $15.67 \%$ & $13.24 \%$ & $2.89 \%$ & $3.64 \%$ \\
117 & $16.45 \%$ & $11.79 \%$ & $4.66 \%$ & $5.22 \%$ \\
104 & $18.99 \%$ & $13.95 \%$ & $11.57 \%$ & $13.28 \%$ \\
103 & $25.83 \%$ & $22.11 \%$ & $6.58 \%$ & $9.20 \%$ \\
112 & $31.32 \%$ & $18.09 \%$ & $11.67 \%$ & $9.58 \%$ \\
\hline
\end{tabular}

how to select a limited number of signatures by a given set of $N$ genuine signatures for finding the most robust stability regions for a subject. We have evaluated the effectiveness of the proposed definition of stability by performing a signature verification experiment based on a standard protocol and using two standard datasets of online signatures.

The results in Table 1 show that our method ranks 5th in terms of $E E R_{\text {avg }}$, but it is the top performing one in terms of $S D_{\text {avg }}$. These results confirm the ability of our stability regions to capture the distinctive aspects of signatures and suggest that, despite the simple classification rule, they are a viable tool for implementing a good performing system for automatic signature verification.

Our future research will focus on adopting more sophisticated classification schemes to improve the system performance, on investigating the behavior of stability regions in case of disguised writers, and on measuring the performance of the proposed method in such a case and when only genuine signatures are available for training.

\section{References}

1. Senatore, R.: The role of Basal ganglia and Cerebellum in Motor Learning: A computational model. PhD Thesis, University of Salerno (March 2012)

2. Raibert, M.H.: Motor control and learning by state space model. AILab. MIT (1977)

3. Impedovo, D., Pirlo, G.: Automatic signature verification: the state of the art. IEEE Transactions on Systems, Man, and Cybernetics, Part C: Applications and Reviews 38(5), 609-635 (2008)

4. Impedovo, D., Pirlo, G., Plamondon, R.: Handwritten Signature Verification: New Advancements and Open Issues. In: 2012 International Conference on Frontiers in Handwriting Recognition (ICFHR), pp. 367-372. IEEE (September 2012) 
5. Dimauro, G., Impedovo, S., Modugno, R., Pirlo, G., Sarcinella, L.: Analysis of stability in hand-written dynamic signatures. In: Eighth International Workshop on Frontiers in Handwriting Recognition, pp. 259-263. IEEE (2002)

6. Huang, K., Yan, H.: Stability and style-variation modeling for on-line signature verification. Pattern Recognition 36(10), 2253-2270 (2003)

7. Marcelli, A., Fuschetto, S.G., Parziale, A.: Modeling Stability in On-line Signatures. In: Proceedings of IGS 2013, pp. 135-138 (June 2013)

8. Itti, L., Koch, C., Niebur, E.: A model of saliency-based visual attention for rapid scene analysis. IEEE Transactions on Pattern Analysis and Machine Intelligence 20(11), 1254-1259 (1998)

9. De Stefano, C., Marcelli, A., Santoro, A.: On-line cursive recognition by ink matching. In: Phillips, J.G., Rogers, D., Ogeil, R.P. (eds.) Proceedings of IGS 2007, pp. 23-37 (2007)

10. De Stefano, C., Garruto, M., Lapresa, L., Marcelli, A.: Using strings for on-line handwriting shape matching: A new weighted edit distance. In: Roli, F., Vitulano, S. (eds.) ICIAP 2005. LNCS, vol. 3617, pp. 1125-1132. Springer, Heidelberg (2005)

11. Yeung, D.-Y., Chang, H., Xiong, Y., George, S., Kashi, R., Matsumoto, T., Rigoll, G.: SVC2004: First international signature verification competition. In: Zhang, D., Jain, A.K. (eds.) ICBA 2004. LNCS, vol. 3072, pp. 16-22. Springer, Heidelberg (2004)

12. Kholmatov, A., Yanikoglu, B.: SUSIG: an on-line signature database, associated protocols and benchmark results. Pattern Analysis and Applications 12(3), 227-236 (2009) 